\title{
Unusual Layer-Dependent Charge Distribution, Collective Mode Coupling, and Superconductivity in Multilayer Cuprate $\mathrm{Ba}_{2} \mathrm{Ca}_{3} \mathrm{Cu}_{4} \mathrm{O}_{8} \mathrm{~F}_{2}$
}

\author{
Yulin Chen, ${ }^{1,2,3}$ Akira Iyo, ${ }^{4}$ Wanli Yang, ${ }^{3}$ Akihiro Ino, ${ }^{5}$ M. Arita, ${ }^{6}$ Steve Johnston, ${ }^{1,2}$ Hiroshi Eisaki, ${ }^{4}$ H. Namatame, ${ }^{6}$ \\ M. Taniguchi, ${ }^{5,6}$ Thomas P. Devereaux, ${ }^{1,2}$ Zahid Hussain, ${ }^{3}$ and Z.-X. Shen ${ }^{1,2}$ \\ ${ }^{1}$ SIMES, SLAC National Accelerator Laboratory, Menlo Park, California 94025, USA \\ ${ }^{2}$ Geballe Laboratory for Advanced Materials, Departments of Physics and Applied Physics, Stanford University, \\ Stanford, California 94305, USA \\ ${ }^{3}$ Advanced Light Source, Lawrence Berkeley National Laboratory, Berkeley, California 94720, USA \\ ${ }^{4}$ National Institute of Advanced Industrial Science and Technology, Tsukuba, Ibaraki, 305-8568, Japan \\ ${ }^{5}$ Graduate School of Science, Hiroshima University, Higashi-Hiroshima, 739-8526, Japan \\ ${ }^{6}$ Hiroshima Synchrotron Radiation Center, Hiroshima University, Higashi-Hiroshima, 739-0046, Japan
}

(Received 22 January 2009; published 17 July 2009)

Low energy ultrahigh momentum resolution angle resolved photoemission spectroscopy study on fourlayer self-doped high $T_{c}$ superconductor $\mathrm{Ba}_{2} \mathrm{Ca}_{3} \mathrm{Cu}_{4} \mathrm{O}_{8} \mathrm{~F}_{2}$ (F0234) revealed fine structure in the band dispersion, identifying the unconventional association of hole and electron doping with the inner and outer $\mathrm{CuO}_{2}$ layers, respectively. For the states originating from two inequivalent $\mathrm{CuO}_{2}$ layers, different energy scales are observed in dispersion kinks associated with the collective mode coupling, with the larger energy scale found in the electron ( $n$-) doped state which also has stronger coupling strength. Given the earlier finding that the superconducting gap is substantially larger along the $n$-type Fermi surface, our observations connect the mode coupling energy and strength with magnitude of the pairing gap.

DOI: 10.1103/PhysRevLett.103.036403

PACS numbers: 71.38. $-\mathrm{k}, 74.72 . J t, 79.60 .-\mathrm{i}$

In the search for the mechanism of high- $T_{c}$ superconductivity, the discovery of electron-boson coupling in the form of a dispersion anomaly with sharp energy scales has attracted significant attention [1-6]. Their universal presence in high- $T_{c}$ superconductor families makes them a possible common origin of pairing. The novel four-layer self-doped [7-9] high- $T_{c}$ superconductor $\mathrm{Ba}_{2} \mathrm{Ca}_{3} \mathrm{Cu}_{4} \mathrm{O}_{8} \mathrm{~F}_{2}$ (F0234), on which previous experiments [7] revealed simultaneous presence of both electron $(n-)$ and hole $(p$-)like Fermi surfaces (FSs) and very different superconducting gaps, provides a unique opportunity for investigating the relationship between the bosonic mode coupling and the pairing gap magnitude in the same sample within a single experiment so that many uncertainties can be eliminated. In addition, the unconventional charge association between $\mathrm{CuO}_{2}$ layers revealed by the fine structure of band dispersion also suggests a reexamination of current theory.

Because of the resolution limitation associated with the relatively high energy photons used, previous angle resolved photoemission spectroscopy (ARPES) measurements were not able to resolve the $p$ and $n$ bands well enough for studying their individual energy scales, or to tell whether there exist fine structures in $p$ or $n$ bands, making it difficult to identify the bands' association with specific $\mathrm{CuO}_{2}$ layers [7]. In our recent investigations, with the superior momentum and energy resolution made possible by low energy photons [10], we unambiguously resolve the fine structures of the bands associated with the $p$-doping FSs, which positively identifies the association of the $p$-type $\mathrm{FS}$ with the inner $\mathrm{CuO}_{2}$ layers. Furthermore, the much improved resolution allowed us to reveal that the $n$ and $p$ bands couple to the collective modes independently and anisotropically, with the $n$-band coupling at higher binding energy with larger strength. By comparing the energy difference of the dispersion anomaly between $n$ and $p$ bands to the difference of their superconducting gap magnitude [7], our finding provides evidence for a direct correlation between mode coupling and pairing strength in a single material under identical experimental conditions.

ARPES experiments were performed at two facilities, with the low photon energy $(8.5-10 \mathrm{eV})$ measurements at beam line 9 A of Hiroshima Synchrotron Radiation Center (HiSOR) and medium energy $(22,55 \mathrm{eV})$ measurements at beam line 10.0.1.1 of the Advanced Light Source (ALS). For the F0234 sample, we utilized $8.5-9.5 \mathrm{eV} / 55 \mathrm{eV}$ circularly or linearly polarized light at HiSOR/ALS, and the $\mathrm{Bi}_{2} \mathrm{Sr}_{2} \mathrm{CaCu}_{2} \mathrm{O}_{8}$ (Bi2212) sample was measured at ALS with $22 \mathrm{eV}$ linearly polarized light to manifest the bilayer splitting [11]. At both facilities, measurement pressure was kept $\leq 4 \times 10^{-11}$ Torr, and data were recorded by Scienta $\mathrm{R} 4000$ analyzers at $20 \mathrm{~K}$ sample temperature. The total convolved energy and angle resolution is $10 \mathrm{meV} / 16 \mathrm{meV}$ and $0.2^{\circ} / 0.2^{\circ}$ at HiSOR/ALS.

In Fig. 1, we show photon energy dependent ARPES measurements along the nodal direction that reveal the fine structure in band dispersions, suggesting an unconventional, layer-dependent charge distribution.

Figure 1(a) is the measurement with a photon energy of $9.5 \mathrm{eV}$. Compared to earlier measurements [7], we find that the $p$ and $n$ bands are much better separated due to the 

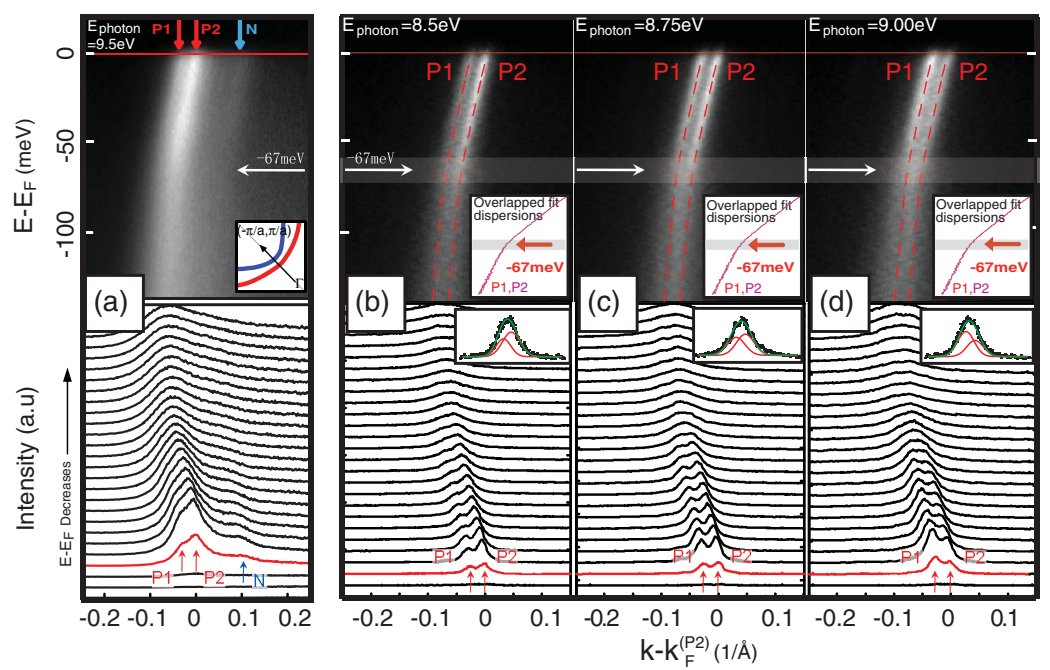

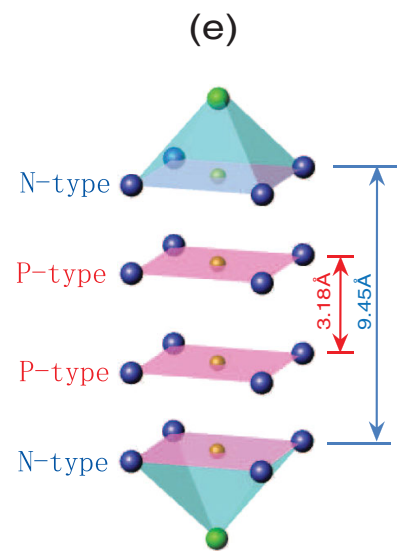

F0234 (f)

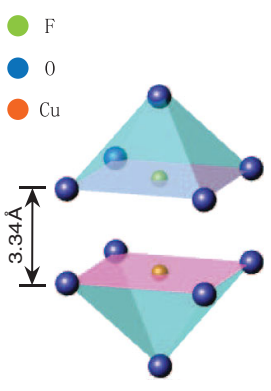

Bi2212

FIG. 1 (color). (a)-(d) Photon energy dependence of ARPES measurement along the nodal direction [indicated by the black arrow in (a) inset]. Upper panels: spectra intensity plots with fitted $p 1-, p 2$-band dispersions (see text) superimposed in plots (b)-(d) and in insets (where the two dispersions are overlapped for comparison); lower panels: MDC stack plots, with an example of two peak MDC fitting at $120 \mathrm{meV}$ binding energy in insets of (b)-(d). (e),(f) Layered structure of $\mathrm{F}_{02} 34$ and $\mathrm{Bi} 2212$ in a unit cell, with the $\mathrm{CuO}_{2}$ layer separations indicated.

better $k$ resolution of the low energy photons [10]. Furthermore, the $n$-band intensity is greatly suppressed at this photon energy, leaving the $p$ band prominent. With these two advantages, we can see indications of fine structure in $p$ band, manifested by the shadow near the main dispersion in the image plot (top) and the clear shoulder of the main $p$-band peak in the momentum distribution curve (MDC) stack plot (bottom). Also in the $p$-band dispersion is a visible "kink" indicated by a sudden electron velocity $(d E / d k)$ reduction, which results naturally from the renormalization of many-body effect as also found in other high $T_{c}$ superconductor families $[1-3,12,13]$. The energy scale of the "kink" is around $67 \mathrm{meV}$, as will be discussed further below. We note here that the two $p$ subbands were predicted by recent LDA calculation [8] but could not be resolved in previous experiments [7].

To further enhance the $p$-band contribution for fine structure study, we chose the photon energy range 8.5$9 \mathrm{eV}$ [see Figs. 1(b)-1(d)], where we not only have comparable subband (noted as $p 1$ and $p 2$ ) intensities, but also almost complete suppression of the $n$-band contribution. The dramatic variation of the $p 1$ and $p 2$ subbands' relative intensities shown in panels (b) to (d) confirms that these two subbands are not trivially the result of the same band of two slightly offset domains on the sample surface. Given that the $p 1$ and $p 2$ subbands are well separated, we can apply the MDC line shape analysis [14] to extract their dispersions, which are superimposed on the (b)-(d) intensity plots and also shown in their insets for clarity. While it is self-evident that two peaks can be fitted at low binding energies, the line shape allows us to do a global fit even to higher binding energies (see inset, for example, at $E_{b}=$ $120 \mathrm{meV}$ ).
The fitted $p 1$ and $p 2$ subbands' dispersions are almost parallel to each other with a $k$ space separation $\Delta k=$ $0.026(1 / \AA)$, which is similar to the bilayer splitting in Bi2212 along the nodal direction, except 3 times larger in magnitude [15]. This anomalously large separation indicates that the interaction between the two $p$-type $\mathrm{CuO}_{2}$ layers is much stronger than that in $\mathrm{Bi} 2212$, thus suggesting that the two $p$-type $\mathrm{CuO}_{2}$ planes are the two inner $\mathrm{CuO}_{2}$ layers and the two outer $\mathrm{CuO}_{2}$ planes are $n$ type, as can be seen in Figs. 1(e) and 1(f): The large separation $(9.45 \AA)$ between the two outer $\mathrm{CuO}_{2}$ layers makes it unrealistic for such strong interaction; on the other hand, the separation $\left(3.18 \AA\right.$ ) between two inner $\mathrm{CuO}_{2}$ layers of F0234 is $0.16 \AA$ less than that in Bi2212 (3.34 $\AA$ ), making the stronger coupling possible. This unconventional charge distribution between inequivalent $\mathrm{CuO}_{2}$ layers, while against the common wisdom [16,17], does agree with the indication from recent NMR observations [9].

Furthermore, Figs. 1(b)-1(d) also indicate an energy scale associated with the dispersion kink at $E_{b}=67 \mathrm{meV}$, with the electron velocity reducing factor $\lambda^{\prime} \sim 0.75$ (see text below for more details). As we will see below, this is much different from that in the $n$ band.

To extract the dispersion and energy scales of $n$ band, medium photon energy $(55 \mathrm{eV})$ was used for intensity enhancement. In Figs. 2(a)-2(c), we show three measurements parallel to the nodal direction. At this photon energy, although both bands have comparable intensity at the node, $n$-band gains weight quickly when moving away from the node. In all three measurements, the simultaneous presence of $p$ and $n$ bands is self-evident, except now the fine structure of $p$ band cannot be resolved due to $k$-resolution limitations [10]. It is obvious from the plots 


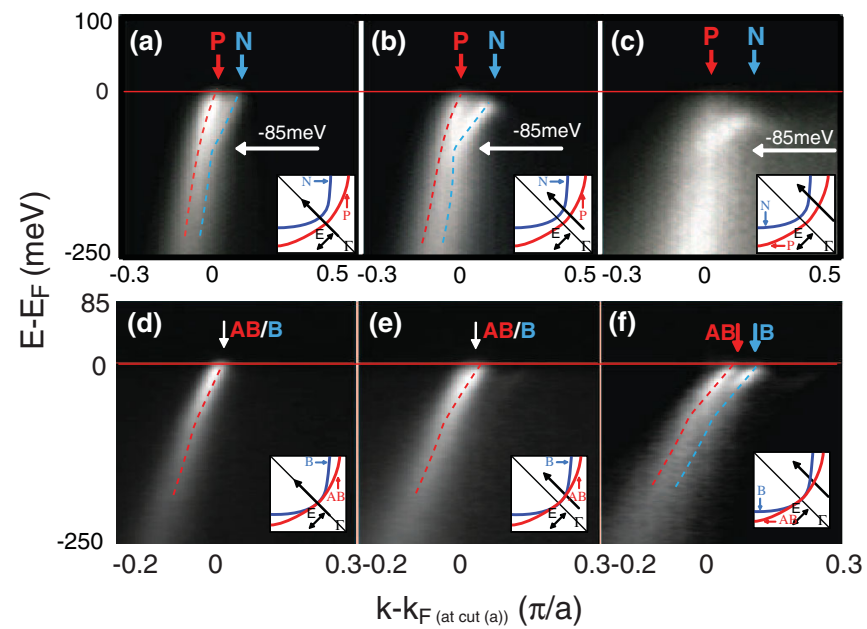

FIG. 2 (color). ARPES measurements on F0234 and Bi2212 parallel to the nodal direction ( $k$ space loci in insets). (a)(c) Spectral intensity plots of F0234 [with fitted $p$ and $n$ dispersions superimposed in (a),(b)]. White arrows at $E_{b}=$ $85 \mathrm{meV}$ indicate the binding energy of the $n$-band dispersion kink. (d)-(f) Measurements at the same $k$ space loci of Bi2212. Fitted dispersions for bonding (B) and antiboding (AB) bands are superimposed on all three plots (see text).

that there exists a clear energy scale indicated by strong $n$-band dispersion kink $(\sim 85 \mathrm{meV})$, which becomes clearer from panels (a) to (c), where $n$ band becomes more dominant. Further, the renormalization of $n$ band also becomes stronger, especially in panel (c), where the $n$-band kink is so prominent that it appears to intersect into the $p$-band dispersion. This clearly shows a banddependent renormalization effect.

This band (or FS) dependence of the mode coupling is different from other high $T_{c}$ superconductors that possess multiple FSs such as Bi2212. For comparison, we illustrate in panels (d)-(f) three measurements for overdoped $\left(T_{c}=\right.$ $70 \mathrm{~K}) \mathrm{Bi} 2212$ sample at the same $k$ space loci. Unlike the large splitting between $p$ and $n$ bands even along nodal direction in F0234 [panel (a)], from (d) one sees that the antibonding $(\mathrm{AB})$ and bonding $(\mathrm{B})$ bands of Bi2212 are almost degenerate. The comparison is similar between panels (b) and (e). In (f), although the $\mathrm{AB}$ and $\mathrm{B}$ bands are now separated, the two dispersions are nearly parallel with no noticeable renormalization difference, which resembles the $p 1$ and $p 2$ bands in Fig. 1 but contrasts obviously to panel (c).

To better compare the electron-bosonic mode coupling strength of both bands, we show in Fig. 3(a) fit dispersions of four fine measurements around the nodal region. (The fitting function used is two Lorentzian peaks convolved with a Gaussian resolution function. As seen in Fig. 2(c), in the region far away from the node, the $n$ band gets too dominant to permit reliable MDC fitting for the $p$ band.) From Fig. 3(a), it is clear that for both $p$ band (warmer color) and $n$ band (cooler color), although the high binding

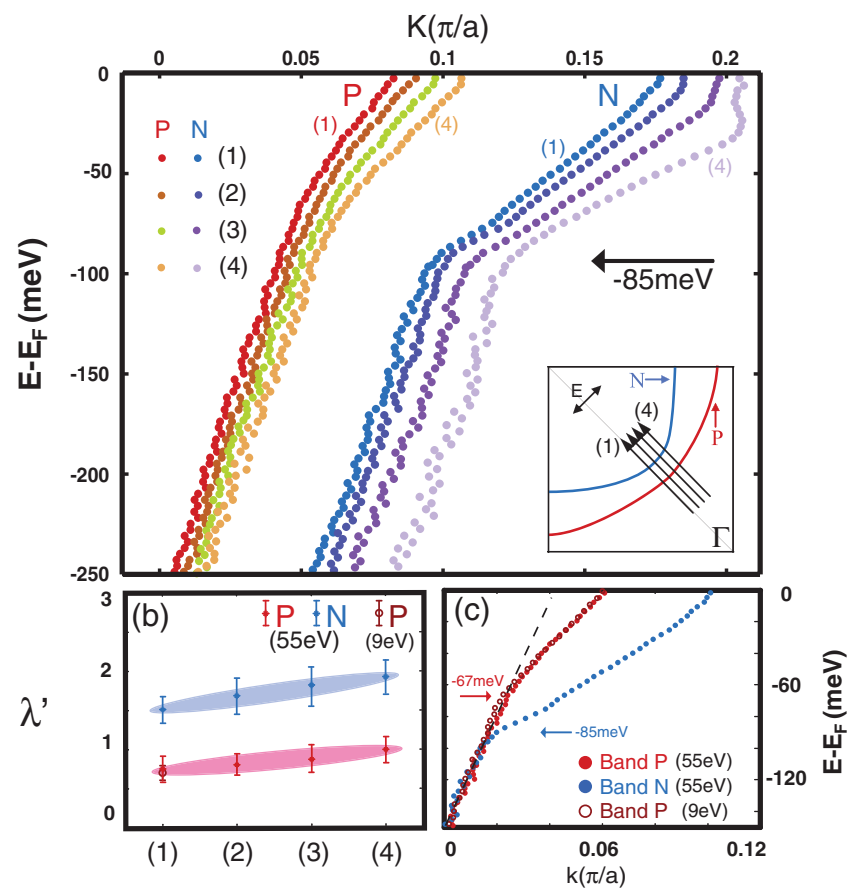

FIG. 3 (color). (a) Dispersions from four sequential cuts with the $k$ space separation between adjacent cuts as $0.05 \pi / a$ (see inset). Warm and cool colors are used for $p$ - and $n$-band dispersions, respectively. (b) Velocity reduction factor $\lambda^{\prime}$ (see text) extracted from the dispersions in panel (a) and in Fig. 1. (c) Overlapped dispersions of $p$ and $n$ bands at nodal direction. The black dashed line is a guide to the eye that shows the coincided dispersions at high binding energy.

energy dispersions are almost parallel, at low binding energy, the electron velocity decreases clearly from cut (1) to (4). This shows that the electron-bosonic coupling is anisotropic in $k$ space for both bands. To quantify the different strength of the renormalization, we calculate the velocity reduction factor $\lambda^{\prime}$ from each dispersion curve [see Fig. 3(b)], where $\lambda^{\prime}$ is defined by $d E / d k_{E b>100 \mathrm{meV}}=$ $\left(1+\lambda^{\prime}\right) d E / d k_{E b<60 \mathrm{meV}}$. The 100 and $60 \mathrm{meV}$ are chosen to avoid the possible uncertainty of kink positions within this region. While $\lambda^{\prime}$ is not exactly the coupling constant $\lambda$, it is proportional to $\lambda$ and thus is also appropriate for comparing the renormalization strength [3]. Figure 3(b) confirms that the mode coupling strength is band dependent and anisotropic in $k$ space within each band. Given that the superconducting gap magnitude for $n$ band is much larger than that of $p$ band [7], the stronger coupling indicated by larger $\lambda^{\prime}$ correlates with larger pairing strength.

Furthermore, in Fig. 3(a), the sharp kink of $n$ band reveals an energy scale of $E_{b}=85 \mathrm{meV}$ (the $p$-band dispersion kink seems blurred due to the presence of strong $n$ band in vicinity and limited $k$ space resolution), which obviously differs from the energy scale of $p$ band $(67 \mathrm{meV}$, see Fig. 1). This difference can clearly be seen by overlapping the $p$ - and $n$-band nodal dispersions in Fig. 3(c). 


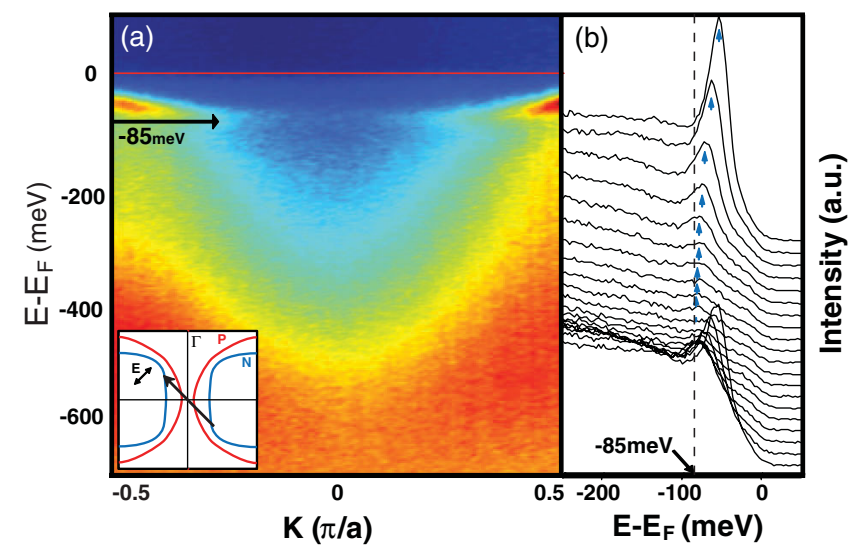

FIG. 4 (color). An ARPES measurement on F0234 cutting through the antinodal point [see inset in (a)]. (a) Intensity plot, the black arrow indicates the binding energy $(85 \mathrm{meV})$ to which the quasiparticle band approaches asymptotically. The bare and quasiparticle bands are originated from the $n$ band (see text). (b) Energy distribution curve (EDC) stack plots of the same measurement. Blue arrows are guides to the eye that trace the sharp EDC peak dispersion. Black dashed lines indicate the $85 \mathrm{meV}$ binding energy positions.

To confirm that the energy scale of the $n$ band does not result from the artifact of fitting procedure due to the presence of two bands, we show a medium energy $(55 \mathrm{eV})$ measurement around the antinodal region in Fig. 4, where $p$-band intensity is almost completely suppressed. From both intensity and EDC stack plots, we see clearly the evidence of electron-bosonic mode coupling [18-20]: starting from low binding energy, a band with sharp EDC peak disperses asymptotically towards the characteristic energy scale $(85 \mathrm{meV})$, and at high binding energy, a band of broad hump traces the (overdamped) bare band dispersion. The fact that the asymptotic energy of the sharp band resides at the same binding energy as the dispersion kink in nodal direction [21-23] proves that the $85 \mathrm{meV}$ energy scale discovered for the $n$-type state is real.

The different energy scales of the $p$ and $n$ bands seen in this compound are rather unusual $[3,24]$. Since the energy scale indicated by dispersion anomaly reflects the addition of bosonic mode energy and the maximum magnitude of $d$-wave superconducting gap: $E_{\text {kink }}=E_{\text {mode }}+\Delta$ [21-23], the $E_{\text {kink }} s$ acquired above (67/85 meV for $p / n$ bands) and $\Delta s$ from previous work (leading edge gap $22 / 42 \mathrm{meV}$ for $p / n$ bands) [7] determine the mode energy to be $45 / 43 \mathrm{meV}$ for $p / n$ bands, respectively. These similar mode energies indicate that the modes coupled to the inner and outer $\mathrm{CuO}_{2}$ layers can either be different modes with similar energies, or even the same mode if the experimental uncertainty is taken into consideration [25]. We encourage future works to study how these modes with similar energies can couple to different types of $\mathrm{CuO}_{2}$ layers and result in vastly different local superconducting gaps.
The unconventional charge distribution between inequivalent $\mathrm{CuO}_{2}$ layers, together with the interplay between the electron-boson coupling strength and the energy gaps of $p$ and $n$ bands also provide excellent tests for theory. The fact that there are two oppositely doped superconducting subsystems in a same material enables a comparison of results from different subsystems under identical experimental and material conditions. The unusual charge distribution indicates an unusual chemical environment in this material and the positive correlation between the gap size and kink strength raises the intriguing question of whether the difference in the local superconducting gap size is linked to the difference of local electron-boson coupling strength.

This work is supported by DOE-BES, DMS\&E at SLAC (DE-AC02-76SF00515) and ALS (DE-AC0205CH11231).

[1] P. V. Bogdanov et al., Phys. Rev. Lett. 85, 2581 (2000).

[2] P. D. Johnson et al., Phys. Rev. Lett. 87, 177007 (2001).

[3] A. Lanzara et al., Nature (London) 412, 510 (2001).

[4] T. K. Kim et al., Phys. Rev. Lett. 91, 167002 (2003).

[5] A. D. Gromko et al., Phys. Rev. B 68, 174520 (2003).

[6] T. Cuk et al., Phys. Rev. Lett. 93, 117003 (2004).

[7] Y. Chen et al., Phys. Rev. Lett. 97, 236401 (2006).

[8] W. Xie et al., Phys. Rev. Lett. 98, 047001 (2007).

[9] S. Shimizu et al., Phys. Rev. Lett. 98, 257002 (2007).

[10] Assuming $\delta k$ and $\delta A$ represent the $k$ space and angle resolution, given the photoelectron energy $E$ and mass $m_{e}$, $\delta k=\delta A \sqrt{2 m_{e} E}$ (nonrelativistic case). Thus for the angle resolution $\delta A$ (fixed for a given analyzer), the $k$ space resoltuion $\delta k$ becomes smaller (better) with lower $E$.

[11] P. V. Bogdanov et al., Phys. Rev. Lett. 89, 167002 (2002).

[12] A. Kaminski et al., Phys. Rev. Lett. 86, 1070 (2001).

[13] S. V. Borisenko et al., Phys. Rev. Lett. 90, 207001 (2003).

[14] T. Valla et al., Science 285, 2110 (1999).

[15] T. Yamasaki et al., Phys. Rev. B 75, 140513 (2007).

[16] Y. Tokunaga et al., Phys. Rev. B 61, 9707 (2000).

[17] H. Kotegawa et al., Phys. Rev. B 69, 014501 (2004).

[18] S. Engelsberg and J.R. Schrieffer, Phys. Rev. 131, 993 (1963).

[19] M. R. Norman et al., Phys. Rev. Lett. 79, 3506 (1997).

[20] M. R. Norman and H. Ding, Phys. Rev. B 57, R11 089 (1998).

[21] M. Eschrig and M. R. Norman, Phys. Rev. B 67, 144503 (2003).

[22] A. W. Sandvik et al., Phys. Rev. B 69, 094523 (2004).

[23] T. P. Devereaux et al., Phys. Rev. Lett. 93, 117004 (2004).

[24] A. Damascelli, Z. Hussain, and Z.-X. Shen, Rev. Mod. Phys. 75, 473 (2003).

[25] Given the breadth of the kink and the previous finding of the presence of multiple modes [X. J. Zhou, Phys. Rev. Lett. 95, 117001 (2005)], these two mode energies are almost identical (even though their absolute values may vary with gap criteria). 